\title{
Der tägliche Asperger
}

Alessia Schinardi

Dr. med., Fachärztin für Psychiatrie und Psychotherapie sowie Kinder- und Jugendpsychiatrie, Mitglied FMH

\begin{abstract}
Autismus-Spektrum-Störungen, die durch Störungen der sozialen Interaktion, Kommunikation und ein enges Interessensgebiet gekennzeichnet sind, werden zunehmend sowohl beim Kind als auch beim Erwachsenen diagnostiziert. AspergerSymptome sind heutzutage neben Burnout-Symptomen die häufigsten Symptome in der Psychopathologie des männlichen Alltags. Kein Mann wird verschont.
\end{abstract}

Ich erhalte einen Telefonanruf eines Kollegen. Er informiert: «... kurz gesagt, die Patientin hat sich gut erholt: Bei mir spricht sie aber nicht, lassen Sie sie auf Italienisch sprechen, so wird sie vielleicht aufgeschlossener und lässt sich dazu überreden, das Antidepressivum wieder zu nehmen ...»

Es ist die übliche Mogelpackung: Meine Kollegen überweisen mir ihre Patienten, ohne sie zurückzunehmen. Aber sie wollen keine von mir übernehmen. Natürlich sind die Patienten glücklicher, wenn sie in ihrer Muttersprache sprechen können ...

In der Tat, die junge Frau lächelt mich an und dankt mir, dass sie kommen durfte, aber es wäre nicht nötig gewesen, sie habe eigentlich gar nicht kommen wollen, es werde schon wieder werden ... Sie erzählt, dass mein Kollege ihr geholfen habe, das Mobbing am alten Arbeitsplatz zu überwinden, schweigt aber darüber, dass sie das Antidepressivum abgesetzt hat.

Sie sagt: «... dann weiss ich nicht, wieso ich einen Rückfall erlitten habe ... könnte es sein, dass Antidepressiva abhängig machen?» Dabei schaut sie mich fragend an.

Durch meinen Blick gebe ich ihr zu folgendes zu verstehen: "... nachdem du die Antidepressiva ohne Absprache mit dem Arzt gestoppt hast, wundert mich nichts - und nein, sie machen nicht abhängig."

Sie spricht weiter.: "Aber es geht besser, ich bin froh, mit einer Frau sprechen zu dürfen, denn ich glaube nicht, dass ich den Mut gehabt hätte, mit dem anderen Psychiater über meinen Mann zu sprechen, wissen Sie, er war so nett ...»

«Von wem sprechen Sie jetzt?», frage ich.

«Ihren Kollegen meine ich. Wir haben nie über meinen Mann gesprochen, aber in letzter Zeit hat er mich zum Weinen gebracht ..."

«Erzählen Sie jetzt während Sie weinen, und antworten Sie bitte auf meine Fragen, ohne zu denken, dass sie ‘komisch`sind. Wir Psychiater sind berühmt dafür, dass wir seltsame Fragen stellen ...»
Aus der Krankenakte sehe ich, dass ihr Mann Informatiker in einem multinationalen Unternehmen ist. "Nichts ist passiert, alles wie vorher ... Nur dass ich in letzter Zeit viel weinen muss ... Aber es geht besser ..." «Erzählen Sie mir bitte, was Sie beschäftigt», ermutige ich sie nochmals.

«Nichts - es tut mir leid ... Eine kleine Dummheit, aber ich wurde wütend auf meinen Mann, weil er eine Szene vor Freunden gemacht hat, die uns ihre Wohnung untervermieten wollten.»

«???»

«Er fing an zu schreien, dass er nicht umziehen wolle, lieber tot sein wolle als provisorisch umziehen, er würde nur in eine Eigentumswohnung ziehen ... Die Freunde wussten nichts von seiner Allergie gegen Veränderungen, und sie waren über seine Reaktion schockiert ... Ich hätte mich am liebsten in den Boden verkrochen! Kurz gesagt, er machte aus einem freundlichen Angebot ein grosses Theater. Wir alle versuchten, das Thema zu wechseln, aber ich schämte mich zu Tode, ich fühlte mich wie eine muslimische Frau, die kein Recht hat zu sprechen. ‘ $\mathrm{Du}$ kannst machen was du willst, hat er mich vor den Freunden angeschrien, so als ob ich eine Fremde sei. Ich hätte stattdessen gerne über das Angebot nachgedacht. Sie erwarteten keine unmittelbare Antwort. - Ist daran etwas falsch?»

Also geht es um das übliche selbstsüchtige Verhalten wie bei vielen anderen Männern, denke ich.

«Und dann?»

"Wir sind so schnell wie möglich heimgegangen.»

«Haben Sie darüber miteinander geredet?»

«Es ist erst vor ein paar Tagen passiert. Ich habe nichts mehr von ihnen gehört, aber ich denke unsere Freunde werden sich nicht so schnell mehr bei uns melden.»

«Ich meine, haben Sie und Ihr Mann darüber gesprochen?»

"Um Himmels willen! Es gibt keinen Dialog ... Ich habe nicht die Kraft, mich ihm zu stellen! ... Aber ich habe mir 
vieles beigebracht ... Wir sind immer allein, und ich habe gelernt, bestimmte Themen zu vermeiden ...»

"Geht es ihm gut?»

"Sicher, das sage ich meiner Schwiegermutter ständig: Schau, wie gut er aussieht! Selbst wenn er sich immer beklagt. Er ist nicht deprimiert, aber er findet immer etwas gegen mich oder findet überall Mängel ... Und das macht mich depressiv!»

«Also, er merkt nicht, wie Sie sich fühlen, er ist nicht empathisch, kann sich nicht mit Ihnen identifizieren?» "Das wäre wirklich zu viel verlangt! Ich habe mich immer als ein offenes Buch beschrieben, zumindest sagen mir alle das, alle ausser ihm ... Er kann sich stundenlang über die Welt beklagen, ohne zu merken, dass ich dabei Selbstmordgedanken bekomme ...»

«Erzählen Sie mir mehr von ihm, seinen Interessen zum Beispiel.»

"Seine Mutter sagt immer, dass das sein erstes Wort ‘Auto〉 gewesen sei. Er hat eine grosse Sammlung der Zeitschrift ‘Sports Cars', seit 1990 und er kennt die Preise aller neuen und gebrauchten Autos auf dem europäischen Markt auswendig. Ich habe das zufällig entdeckt. - Ist das normal?»

«Liest er auch anderes?»

"Abgesehen von diesem Magazin und Fachbüchern, nein ... Aber er sieht wirklich gern fern.»

«Was mag er im Fernsehen?»

«Ein wenig von allem, aber mehrheitlich zappt er.» «??»

«Er ändert schnell den Kanal, ohne anzuhalten, dabei muss ich weggehen ... Ich bekomme Kopfschmerzen, aber ich vermute, er geniesst es. - Ist das normal?»

"Sagen Sie mir, ob ich es richtig verstanden habe. Aber davor putzen Sie sich die Nase und trinken Sie das Glas Wasser, denn vielleicht fliessen noch mehr Tränen: Also Ihr Ehemann ist ein erfolgreicher IT-Manager, hat nur wenige Interessen und das nur in bestimmten Bereichen. Sie sind ein kinderloses Paar. Sie fühlen sich einsam und missverstanden, während er so glücklich zu sein scheint.»

"Genau! Ich bin es, bei der etwas falsch ist ... Aber vielleicht ist nicht alles meine Schuld ..."

Während meine rechte Hemisphäre Empathie zeigt, öffnet meine linke Hemisphäre die Schublade für Asperger, die der hochfunktionalen Autististen.

«Bitte brauchen Sie bitte keine Worte wie «richtig〉 oder (falsch), «normal» oder «handicapiert) - bitte -, denn das sind Tabuwörter für uns Psychiater!»

"Was denn?"

«Erzählen Sie bitte weiter, dann erkläre ich es Ihnen ... Erzählen Sie mir bitte von Ihren gemeinsamen Freunden, von dem, was Sie in Ihrer Freizeit machen.» «Einmal im Jahr gibt es ein berufliches Abendessen und dann sehe ich, dass seine Kollegen ihn schätzen, auch wenn sie ihn «lapidar nennen. Bei diesen Gelegenheiten wurde mir klar, dass er selbst nie von sich und seiner Arbeit plaudert, selbst wenn er die Ausrede braucht, es sei langweilig, über industrielle Programmierung zu sprechen. Seine Kollegen jedoch tun es und machen ihren Beruf auch für Laien wie für mich interessant ... Mein Mann sagt, dass er in seiner Freizeit nicht mit Arbeitskollegen verkehren will, und selbst darin, keine andere Freunde zu besuchen, ist er unnachgiebig, wie in allem ... auch weil er keine Zeit hat - wir keine Zeit haben. Wir haben ja keine gemeinsamen Freunde! Wir sind immer alleine.» «Er hat also keine Freunde, sondern Kollegen: Kann er eine schöne Erfahrung mit anderen teilen? Zum Beispiel das neue Auto?»

"Auf gar keinen Fall! Ich muss den Schwiegereltern alles selber berichten. Wissen Sie, er wollte das neue iPhone nicht in die Heimat mitnehmen, mit der Ausrede, dass für Telefonate in Italien immer noch das alte Natel benutzt werden müsste. Aber ihm geht es nicht nur darum, dass er sich nicht zur Schau stellen möchte, ich habe eher den Eindruck, dass er nicht möchte, dass andere seine Sachen anfassen ..."

«Es scheint, dass ... er wenig Freude daran hat, etwas zu teilen, aber kann er etwas leihen?»

"Leihen, Geben und Überraschungen sind keine Wörter, die zu seinem Vokabular gehören ...»

"Ich nehme an, er ist sehr ordentlich ...»

«Nur für seine Sachen ... seine Sachen sind nur seine, und er hält sie in einer religiösen Ordnung! Lange dachte ich, er wäre davon besessen! Meine Sachen gehören stattdessen uns beiden!»

«Sagen Sie mal, kann er Witze erzählen?» «Wie bitte?»

«Ich sagte ja, wir Psychiater können seltsame Fragen stellen: Kann Ihr Mann Witze erzählen? Und falls ja, mögen Sie sie?»

«Eigentlich kann ich mich über all diese Jahre nicht erinnern, dass er je einen erzählt hat: Er hat sogar Schwierigkeiten, Gesellschaftsspiele zu spielen, die paar Male beobachtete er den Rest der Familie ... aber ... er plappert meine Sätze nach wie ein Papagei! Ich erinnere mich, dass meine Cousine einmal beruflich ein Konzert der Pooh besuchen musste. Die junge Dame konnte die Popband nicht ausstehen... Kurz gesagt, um sie zu trösten, sagte ich ihr, dass die Pooh unsere Rolling Stones seien ..." «?!»

"Ich weiß nicht, wie Sie über die Poohs denken, aber als mein Mann das hörte, lachte er sich dabei den Bauch voll, er wiederholte den Witz bis zum geht nicht mehr, und am nächsten Tag sagte er mir: ‘Dann sind die Ricchi e Poveri unsere Abba! - Und das er wiederholte er immer wieder ...” 
"Auch als Sie nicht mehr lachten... - Sie sagen, dass er keine Witze erzählen kann. Aber er hat Spass, wenn jemand anderes Witze erzählt?»

"Sagen wir, wenn er lacht, sind es krasse Witze ... Und jetzt, wo ich daran denke - wenn er lacht, wird er übertrieben laut, sehr laut, bis er Tränen lacht ... fast respektlos. Aber ich empfinde das vielleicht auch nur so, weil es so selten ist, ihn glücklich zu sehen."

«Lassen Sie mich eine weitere Zusammenfassung machen: Ich habe aus Ihrer Geschichte verstanden, dass Ihr Mann wenig soziale Fähigkeiten hat: Er verkehrt in seiner Freizeit mit niemandem, er spricht nicht über sich selbst oder über seine Arbeit, er hat Schwierigkeiten zu verstehen, wie sich sein Gegenüber fühlt. Sie sagen, er sei nicht flexibel, aber das sei ihm egal, er sei glücklich so ...»

"Dass er keine flexible Person ist, stimmt völlig. Wir machen immer, was er will! Wenn ich etwas tun will, womit er nicht einverstanden ist und ich ihn nicht überzeugen kann, fängt er an zu schreien ... Ich muss ihn vor vollendete Tatsache stellen und er verzeiht mir nie ..." «Zum Beispiel?»

«... habe ich vor Jahren versucht, ihn davon zu überzeugen, ein Musical zu besuchen. Er begann zu schreien, er hasse Musicals. Punkt. Ich kaufte zwei Tickets und sagte ihm, dass, wenn er mich nicht begleiten würde, ich mit einem Freund gehen würde. Daraufhin kam er mit. - Die Geschichte mit dem Umgang mit Geld ist noch eine andere. - Auf jeden Fall, das Musical erwies sich als Fiasko und er beschwerte sich monatelang darüber. - Ich ergreife keine Initiativen mehr.»

«Eben. Reden wir über euch zwei. Nehmen Sie bitte ein neues Kleenex. Wie ist die Beziehung zwischen Ihnen beiden? Sie sind jetzt 10 Jahre verheiratet. Sieht er Ihnen in die Augen?»

"Ja, das tut er, aber wenn ich zum Beispiel einen Bekannten auf der Straße treffe, starrt er plötzlich in das nächste Schaufenster. Mir war das zuerst peinlich, jetzt ist es mir egal ..."

«Hat er Schwierigkeiten, Fremde anzusprechen?» "Er verirrt sich stundenlang, bevor er nach dem Weg fragt! Im Restaurant lässt er mich bestellen und wehe, wenn ich einen Fehler mache! Anstatt mit dem Kellner zu sprechen, streitet er mit mir!»

«Geben Sie mir bitte ein Beispiel.»

"Wir waren in Frankreich und ich bestellte ein gut durchgebratenes Steak für ihn. Sobald der Kellner wegging, tadelte er mich heftig, anstatt dem Kellner zu sagen, dass er das Fleisch rosa haben wollte."

«Erzählen Sie mir noch weitere spezielle Dinge, die Sie irritieren.»

"Ich liess das Bügeleisen auf das Parkett fallen. Er schimpfte deswegen monatelang, obwohl ihm auch klar war, dass ich es nicht absichtlich getan hatte ... Ich fühlte mich wie ein Kind. Er wollte nicht aufhören ...» «Wann hört er auf?»

"Wenn ich gehe ... Ich gehe oft in den Keller, zur Waschmaschine ..."

«Merkt er nicht, wie es ihnen geht?»

«Er kann stundenlang darüber reden, was ihn interessiert und merkt nicht, dass es mich langweilt... Manchmal kommt er auf mich zu und reibt mir Dinge unter der Nase, die er mir zeigen will, fast immer ein neues Auto ... Er folgt mir aufs WC, so dass ich die Tür abschliessen muss. Irgendwann sagte ich ihm direkt, er solle sich nicht an WC-Tür anlehnen, wenn ich drin bin... Zuerst dachte ich, es wäre Liebe ... Dann dachte ich über andere Hypothesen nach ...» «Zum Beispiel?»

"Dass er verwöhnt ist und sofort die ganze Aufmerksamkeit will! Egozentrisch sagen die Therapeuten, oder? ... Ich weiss, dass er mich liebt, auch wenn er es mir nicht sagt. Aber es ist, als hätte er Schwierigkeiten damit, dass wir zu zweit sind.»

"Also, Sie versuchen Ihn zu verstehen, aber er versteht Sie nicht, nicht einmal nach so vielen Jahren ...» "Manchmal fühle ich mit wie E.T., eine Ausserirdische." «Erzählen Sie mehr davon...»

"Zum Beispiel will er, dass ich eine genaue Sprache anwende, er klammert sich an Worten fest, meiner Meinung nach, um Zeit zu gewinnen. ... Als ich einmal Spaghetti kochte, bat ich ihn, mir das Sieb zu reichen. Statt Sieb sagte ich anscheinend 'Topf〉. Ich hielt den hiessen Spaghettitopf in den Händen und deutete mit dem Kopf auf das Ding zwischen uns. Es gab da nur das Sieb. Er erstarrte mich an und fragte: ‘welcher Topf?» Später hat er mir erklärt: «Selber Schuld, wenn du dich verbrannt hast. Du hättest doch Sieb sagen sollen!' ... Ich habe andere Beispiele für seinen Perfektionismus, wobei mir das eher als Pedanterie erscheint.»

"Könnte es sein, dass Ihr Ehemann Schwierigkeiten hat, eine Situation zu verstehen, die nur aus Gesten besteht, wo der Tonfall wichtig ist und wo es nicht in erster Linie um den eigentlichen Inhalt von Informationen geht?» «Wie bitte?»

«Es scheint, dass das Zusammenspiel aus Sprache, Gesten und Zeichen, welche ein Paar im Laufe der Zeit voneinander lernt, nicht existiert.»

«Er gibt mir Dinge zum Wegwerfen, obwohl ich mit beiden Händen beschäftigt bin ... Er spricht mit mir, wenn ich den Staubsauger oder den Haartrockner betätige, ist das nicht kindisch?»

Ich schaue auf die Uhr, die Klientin weint immer noch ... Wenn ich ihr am Ende der Sitzung nichts Konstruktives sagen kann, werde auch ich weinen: Ich werde mir vorwerfen, ich hätte alle Ausbildungen über das Anzapfen 
von Ressourcen in Klienten umsonst besucht, es würde keine nächste Sitzung geben: Die Klientin kehre nach Hause zurück und werfe sich aus dem Fenster ...

Deshalb sagte ich: «Jetzt erzähle ich Ihnen etwas Positives über ihren Mann: Er füllt die Steuererklärung aus und er kann Haushaltgeräte reparieren. Stimmt das?» "Woher wissen Sie das?»

«Und er liebt sie sehr, auch wenn er es Ihnen nie gesagt hat.»

"Aber wie viele Jahre wird es noch dauern, bis er von uns beiden im Plural spricht? Bevor er meine schwere Tasche trägt, mir Wasser reicht, für uns beide in Restaurant bestellt?»

Jetzt weine ich auch ...

"Entschuldigen Sie, ich sollte über mich und nicht über ihn reden. Ich weiss genau, dass man die Abwesenden nicht ändern kann, das weiss ich von ihren Vorgängern." "Genau, liebe Frau X, ich bin auch verhaltenstherapeutisch orientiert, aber die Zunge schlägt, wo der Zahn wehtut ... und ich möchte, dass Sie Ihren Frust abladen ...»

Meine Gedanken rattern. Wenn ich ihr verallgemeinernd sage, dass viele Ehemänner so sind, bringt sie sich um. Wenn ich aber sein Verhalten mit dem breiten und weiten Spektrum zwischen Normalität und extremer Männlichkeit bis zu Autismus relativiere, bringt sie mich um ...
Deshalb sage ich ihr: «Liebe Frau X, wenn Sie sich scheiden lassen wollten, wären Sie zu einem Anwalt gegangen! Sie möchten ihren Ehemann aber nicht loswerden. Ich kann Ihnen verschiedene Techniken zeigen, wie Sie Ihre Belastung ändern können, um sie auf ein erträgliches Mass zu reduzieren ... angefangen mit Ihrer Tasche ...»

«Meine Tasche?»

«Ihre Tasche ist eigentlich ein kleiner Koffer, den Sie überall mitschleppen, auch wenn sie um die Ecke wohnen. Ich kann Ihnen beibringen, das Positive des Dysfunktionalen sehen zu lernen... und es ‘anzupassen` oder eben so leben, wie es für sie akzeptabel ist ...»

«Zum Beispiel?»

«Zum Beispiel ist er pünktlich, er ist vertrauenswürdig, er ist immer bei Ihnen, Sie müssen ihn mit niemandem teilen, er wird sie niemals betrügen ... schon ein einziger Gedanke, die Frau zu wechseln macht ihn krank!»

Die Frau nickt lächelnd.

"Jetzt habe ich sie zum Lachen gebracht - bis zum nächsten Mal.»

Wollen Sie, meine Leserinnen und Leser, meine Diagnosen wissen? Ich darf sie nicht nennen, denn ich bin an die Schweigepflicht gebunden ... 